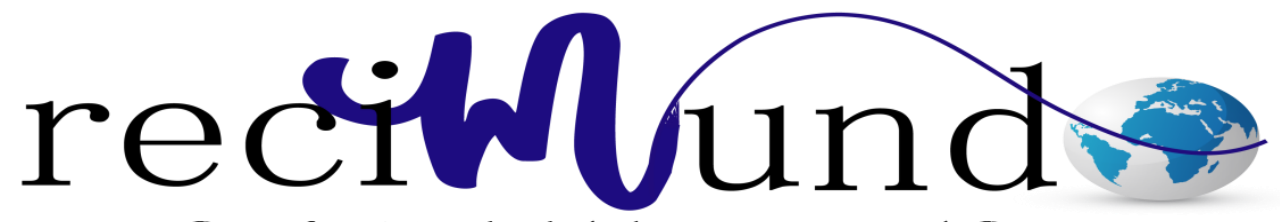

Revista Científica Mundo de la Investigación y el Conocimiento

Eva María de Lourdes Loaiza Massuh ${ }^{\text {a }}$ Jazmin Arce Recalde ${ }^{\text {b; }}$ Mauricio Vicente Villacreses Cobo ${ }^{\mathrm{c}}$

Inteligencia emocional, el desempeño en ventas y compromiso organizacional

Revista Científica Mundo de la Investigación y el Conocimiento. Vol. 1 núm., 5, diciembre, 2017, pp. 578-600

DOI: $10.26820 /$ recimundo/1.5.2017.5578-600

Editorial Saberes del Conocimiento
a. Universidad de Guayaquil; eva.loaizam@ug.edu.ec
b. Universidad de Guayaquil; jazmin.arcer@ug.edu.ec
c. Universidad de Guayaquil; mauricio.villacresesc@ug.edu.ec 


\title{
Inteligencia emocional, el desempeño en ventas y compromiso organizacional
}

Vol. 1, núm. 5., (2017)

Eva María de Lourdes Loaiza Massuh; Jazmin Arce Recalde; Mauricio Vicente Villacreses Cobo

\section{RESUMEN}

El talento humano de una empresa que comprobadamente se encuentra comprometido con los objetivos y valores de su organización, no sólo constituye una ventaja de la empresa sobre otra, sino también como una ventaja competitiva sostenible para muchas de instituciones. Por otra parte el talento humano con un bajo sentido de pertenencia, lealtad y compromiso con la organización, no sólo no se mueve hacia las metas de la organización, sino que además debilita mediante contagio, el espíritu de colaboración interna de una organización, creando de una cultura organizacional de indiferencia a los problemas y preocupaciones de la organización, lo que retrasa el éxito de la organización o evita que se prevean las soluciones a tiempo.

Palabras Claves: Inteligencia emocional; desempeño; ventas.

\begin{abstract}
The human talent of a company that is proven to be committed to the objectives and values of its organization, is not only an advantage of the company over another, but also as a sustainable competitive advantage for many institutions. On the other hand, human talent with a low sense of belonging, loyalty and commitment to the organization, not only does not move towards the goals of the organization, but also weakens through contagion, the spirit of internal collaboration of an organization, creating an organizational culture of indifference to the problems and concerns of the organization, which delays the success of the organization or prevents the foreseen solutions on time.
\end{abstract}

Keywords: Emotional intelligence; performance; sales 
Inteligencia emocional, el desempeño en ventas y compromiso organizacional Vol. 1, núm. 5., (2017)

Eva María de Lourdes Loaiza Massuh; Jazmin Arce Recalde; Mauricio Vicente Villacreses Cobo

\section{Introducción.}

La realidad que el mundo económico de hoy atraviesa una muy marcada etapa de crisis económica y los equipos de vendedores de las empresas, esto es, el corazón económico de las instituciones no están exentos de ser influenciados por el temor que esta crisis infunde produciendo un clima organizacional tenso, poco comprometido, además de un bajo rendimiento laboral, dejando ver la necesidad de desarrollar en sus integrantes la inteligencia emocional. Shooshtarian, Ameli y Lari (2013) concluyeron que la inteligencia emocional es uno de los aciertos más relevantes que la Psicología ha introducido en el mundo comercial en los últimos 10 años y es que según lo aseveraron Goleman, Boyatzis \& Mckee (2002) la inteligencia emocional puede desempeñar un papel positivo en el desarrollo de compromiso organizacional

Según lo aseverado por Seyedeh, Hoda, Forogh \& Azar (2013) la inteligencia emocional es una herramienta útil para predecir el nivel de satisfacción laboral y compromiso organizacional. Chernis y Goleman (2013) expresaron que decenas de entendidos luego que indistintamente llevaron a cabo investigaciones en más de 500 instituciones laborales del mundo, coincidieron al concluir que la Inteligencia Emocional es un factor determinante en el desempeño de cualquier trabajo, así como en el éxito profesional. para Alkahtani (2016) las organizaciones necesitan empleados que estén comprometidos en su trabajo para que puedan contribuir a la supervivencia de la organización en la ardua competencia de mercado, un empleado que pudiera aumentar sus habilidades de inteligencia emocional o reforzar sus capacidades en un contexto de cultura organizacional bien preparada, podría de forma natural identificar sus necesidades de mejor y más equilibrada manera, pudiendo alcanzan una mayor satisfacción, fue por esto que Marcano (2006) recomendó proveer a la fuerza laboral de 


\section{Inteligencia emocional, el desempeño en ventas y compromiso organizacional}

Vol. 1, núm. 5., (2017)

Eva María de Lourdes Loaiza Massuh; Jazmin Arce Recalde; Mauricio Vicente Villacreses Cobo

capacitación en lo referente a la inteligencia emocional para que su productividad se oriente al éxito personal, profesional y a robustecer la conducta organizacional.

Por su lado Amirghodsi y Naeini (2016) expresaron que si el objetivo de una empresa es perseguir que se logre la eficiencia del talento humano, desarrollar un buen nivel de productividad en su trabajo y fortalecer el compromiso organizacional, se debe utilizar los resultados y principios psicológicos. En este sentido, la inteligencia emocional podría proporcionar una conducta organizacional enfocada, un ambiente de trabajo agradable y como resultado la satisfacción en el trabajo de los empleados además de una gestión eficaz y el avance de la organización.

Un alto nivel de inteligencia emocional, no un alto índice de coeficiente intelectual (IQ), aumenta el rendimiento, comprensión estratégica y satisfacción en el trabajo, es de un papel positivo en la colocación y disminuye problemas del empleado. Simons y Simons (2005) narraron las causas de la baja en las ventas y del tenso clima organizacional de una institución en los Estados Unidos y concluyeron que la raíz del problema yacía en que quien estaba a cargo de las ventas tenía un IQ muy alto, pero no poseía el grado correcto de Inteligencia Emocional, esto es, determinadas características que son necesarias para desempeñar un cargo de gestión. La Inteligencia Emocional, la cultura organizacional y el desempeño en ventas presentaron un alto grado de correlación en este caso.

Goleman, Bennett y Barlow (2012) en su búsqueda de establecer diferencias entre un alto nivel de Inteligencia Emocional y alto nivel de IQ, relataron sus impresiones de una investigación que se llevó a cabo entre un grupo de jóvenes trabajadores Estadounidenses que 
Inteligencia emocional, el desempeño en ventas y compromiso organizacional Vol. 1, núm. 5., (2017)

Eva María de Lourdes Loaiza Massuh; Jazmin Arce Recalde; Mauricio Vicente Villacreses Cobo

prestaban sus servicios profesionales en otros países en medio de comportamientos organizacionales variados, en este trabajo se dejó ver que en lo concerniente a Inteligencia Emocional no son los más destacados trabajadores quienes ostentan de un IQ más alto que otros, sino quienes poseyendo un IQ elevado también logran desarrollar las competencias emocionales en medio de una cultura organizacional definida y citaron como ejemplo el campo de las ventas, donde las tensiones características de este tipo de trabajo suelen alterarse con frecuencia y se debe aprender a estudiar las reacciones de los demás y a modular a punto de balance las propias..

Del Henar (2013) explicó que el IQ no está desvinculado de la inteligencia emocional (IE), sino que ambos son aspectos complementarios, ambos son parte intrínseca de las personas, el contraste lo establecen la cantidad y la calidad en los que se los llegue a ampliar. Goleman (1995) anotó que las conquistas o los desaciertos acaecidos en la vida de los seres humanos no estriban absolutamente de cual sea su nivel de IQ o de las conquistas académicas, más del nivel de inteligencia emocional que se haya desarrollado y en el quehacer profesional, en cómo se lleva esta inteligencia emocional alcanzada hasta la práctica en la vida empresarial, en el caso del presente estudio en la práctica llevada a cabo por los equipos de vendedores.

Las fuerzas de ventas de las empresas del mundo actual, en especial las de países con economías emergentes, están con mayor frecuencia sometidos a inestabilidades producidas por factores diversos como los repentinos cambios protagonizados por los gobiernos de turno (Gil, Suárez, \& Pérez, 2013) lo que demanda de mayor forma del adecuado uso de sus capacidades como vendedores de éxito, en medio de este escenario, Boons y Lüdeke-Freund (2013) invitaron a examinar la influencia de los factores internos y externos sobre quienes salen a los mercados en busca de nuevos negocios. 


\section{Inteligencia emocional, el desempeño en ventas y compromiso organizacional}

Vol. 1, núm. 5., (2017)

Eva María de Lourdes Loaiza Massuh; Jazmin Arce Recalde; Mauricio Vicente Villacreses Cobo

Fragoso (2015) destacó que el conocerse a uno mismo, el dominio propio y la motivación personal, determinan el autocontrol, así como también facilitan la factibilidad de hacer uso de manera estratégica e inteligente de las emociones propias. Miranda (2014) por su parte afirmó que la vida de las personas está conformada por una secuencia de acuerdos, lo que demanda el ingresar en una continua transacción en los ámbitos tan diversos donde las personas llevan a cabo sus tareas del día a día y las victorias conquistadas dependen en cómo se lo realice y de cuan comprometida esté con esa labores y con la organización a la que se pertenece. Miranda (2014) destacó también que los equipos de representantes de ventas reciben los impactos de ignorar sus propias capacidades en los campos la negociación, de inteligencia emocional y la comunicación, así como también de la falta de conocimiento de cómo hacer mejor uso de ellas o de llegar a desarrollarlas hasta conquistar un nivel de provecho profesional más amplio y variado, es por esto que Chacón (2013) remarcó, la inminente necesidad de instruir a las fuerzas de ventas en cuanto a la Teoría de la Inteligencia Emocional aplicada al contexto organizacional y entregarles las herramientas que les permitan explotar al máximo sus capacidades hasta ahora desconocidas, concluyó afirmando que esto generaría un seguro compromiso organizacional.

Se hace necesario entonces incursionar en la literatura para definir lo que es: inteligencia emocional, compromiso organizacional y desempeño en ventas, para seguidamente realizar el análisis de la relación que existe entre estas variables.

\section{Revisión de Literatura.}

\section{Inteligencia emocional}


Inteligencia emocional, el desempeño en ventas y compromiso organizacional Vol. 1, núm. 5., (2017)

Eva María de Lourdes Loaiza Massuh; Jazmin Arce Recalde; Mauricio Vicente Villacreses Cobo

Para entender de manera más clara el porqué de las decisiones que las personas tienden a tomar en un entorno laboral y para potenciar aquellas que son buenas y guiar por otro rumbo aquellas que no son tan acertadas, se hace necesario ingresar en el estudio de la Inteligencia Emocional que el campo de la Psicología no ofrece y al realizar la revisión de literatura referente a Inteligencia Emocional, el Desempeño en Ventas y Compromiso Organizacional, se destacó lo siguiente:

Inicialmente Thorndike, Bregman y Cobb (1926) mencionaron que uno de los elementos relevantes de la inteligencia humana era la capacidad que lo hace un ser social, ya que hace posible, la comunicación, el entendimiento, relacionarse y además lo lleva para inspirar a otros. La primera referencia encontrada con relación al tema de Inteligencia Emocional fue la de Gardner (1983) quien manifestó que la conceptualización de la inteligencia personal está constituida por dos constructos: Uno de ellos, el constructo intrapersonal o también llamado inteligencia emocional y el constructo interpersonal o mejor conocido como inteligencia social.

Luego Salovey y Mayer (1990) presentaron su definición de inteligencia emocional como un subconjunto de la inteligencia social que involucra la habilidad de monitorear las emociones y los sentimientos propios y ajenos para diferenciarlos y utilizar esta información para guiar los pensamientos y acciones del individuo, así como el ordenar las emociones de tal manera que mejore la vida. Posteriormente Mayer y Salovery (1997) definieron la Inteligencia Emocional como aquella capacidad desarrollada en las personas que percibe emociones y sentimientos propios y también de otras personas, que accede y genera emociones para ayudar al pensamiento propio y de otros a entender las emociones y el conocimiento emocional y de manera reflexiva ayudar a regular las emociones para promover el crecimiento emocional e 


\section{Inteligencia emocional, el desempeño en ventas y compromiso organizacional}

Vol. 1, núm. 5., (2017)

Eva María de Lourdes Loaiza Massuh; Jazmin Arce Recalde; Mauricio Vicente Villacreses Cobo

intelectual con la finalidad de poder utilizar esta información para guiar a las personas en su forma de pensar y de ejecutar sus ideas. La Inteligencia emocional no solo abarca las emociones, sino también sus aplicaciones en la comunicación humana guiando a la utilización positiva de las emociones (Gholami, Shams, \& Amoozadeh, 2013)

Bar -On (2006) definió la Inteligencia Emocional como la variedad de emociones, capacidades y aptitudes personales y sociales experimentadas por las personas, que les proporcionan un impacto o la capacidad de desafiarse a ellos mismos para enfrentar las demandas y las presiones del medio en una forma eficaz. Otra definición de Inteligencia Emocional es la de Wechsler ( 1958) quien la presentó como la destreza humana que puede ser natural, haber sido desarrollada, agregada o global que tiene como objetivo guiar a la persona a actuar previa determinación de propósitos, a pensar de manera racional y a conducirse eficazmente en su entorno. En otro estudio, se estableció que las emociones son las respuestas psicológicas, expresada en sentimientos subjetivos que están interrelacionadas con actividades de índole intelectual. (Psilopanagioti, Anagnostopoulos, Mourtou, \& Niakas, 2012).

Bande, Fernández-Ferrín, Varela y Jaramillo (2015) presentaron un consenso en cuanto al concepto epistemológico de la Inteligencia Emocional y expresaron que los investigadores estudiados coinciden en definir la inteligencia emocional como una capacidad humana, que puede ser desarrollada y que ontológicamente involucra aquel sentido del conocimiento de las emociones propias y de las ajenas y como las propias pudieran ser reflejadas en torno de los demás en un medio ambiente social específico, en el caso de la presente investigación en un entorno profesional-laboral. 


\section{Inteligencia emocional, el desempeño en ventas y compromiso organizacional}

Vol. 1, núm. 5., (2017)

Eva María de Lourdes Loaiza Massuh; Jazmin Arce Recalde; Mauricio Vicente Villacreses Cobo

Por su parte Saarni (1990), explicó que desde su perspectiva la inteligencia emocional está conformada por ocho constructos interrelacionados y que son habilidades emocionales y sociales. También Bar-On (2000) expuso que la inteligencia emocional es una combinación determinada que busca conseguir un comportamiento humano eficaz, sus componentes son: habilidades, un número de competencias intrapersonal, interpersonal y facilitadores, por lo que concluyó que basado en estos elementos es más preciso llamar a estos constructos inteligenciasocial-emocional y no tan solamente inteligencia emocional.

Mayer, y Salovey (1997) explicaron que la Inteligencia emocional está compuesta por cuatro habilidades: La percepción, el uso, el entendimiento y la regulación de las emociones, también expusieron la teoría respecto a que casi todas las habilidades que están condensadas en la inteligencia emocional se pueden llegar a explayar desde la educación impartida en casa y el entrenamiento académico proporcionado por las instituciones educativas, El desarrolla de la Inteligencia Emocional que inicia en el hogar, está fundamentado con una relación sólida entre padres-hijos. Los padres son las primeras personas quienes identifican, enseñan y dan ejemplo de cuales son las emociones para después respetando las emociones de los hijos, los guían en el cómo hacer uso de ellas en las experiencias que la sociedad presenta. Mayer, Caruso y Salovey (2000) aseveraron que la inteligencia general, es un conjunto de capacidades y procesos entre los que sobresale el razonamiento abstracto, el cual puede ser desarrollado a través de las emociones y a partir de una perspectiva cognitiva o procesando la información referente a las emociones. También Loaiza (2016) explicó que Kafetsios y Zampetakis (2008) plantearon la teoría que personas con una inteligencia emocional desarrollada reportaban mayor satisfacciones laborales, esta teoría establece que los individuos con mayor nivel de Inteligencia emocional, la 


\section{Inteligencia emocional, el desempeño en ventas y compromiso organizacional}

Vol. 1, núm. 5., (2017)

Eva María de Lourdes Loaiza Massuh; Jazmin Arce Recalde; Mauricio Vicente Villacreses Cobo

utilizan en su medio laboral para evaluar y direccionar las emociones de otros, lo que les permite fomentar la interacción interpersonal. Goleman (1995) definió la inteligencia emocional en cuatro dimensiones: autoconciencia, autocontrol, conciencia social y manejo de las relaciones, mismas que posteriormente fueron presentadas por Goleman (2013) como la teoría de Primal Leadership, la cual establece que la inteligencia emocional se subdivide en estas cuatro habilidades y que combinando las mismas se puede llegar a definir las competencias sociales y personales de un ser humano. Mayer y Salovery (1997) consideraron la autoconciencia o saber lo que alguien siente como el primer paso para ser capaz de manejar la interacción con otros. Goleman (1995) estableció que el autocontrol es la habilidad de regular los efectos del estrés como lo son la ansiedad y la ira e inhibir la impulsividad emocional. Bar-On (2000) explicó que la tercera dimensión, conciencia social, incluye la capacidad de sentir, entender y reaccionar a las emociones de los demás. Por otro lado Goleman (1998) delimitó la conciencia social estableciendo que abarca la competencia de la empatía, la cual definió como la capacidad para leer señales no verbales de las emociones negativas, especialmente el enojo y el miedo y a juzgar la confiabilidad de los demás. Se trata de entender los sentimientos del otro, no experimentarlos. También Golemena(1998) definió el cuarto constructo, manejo de las relaciones, implica la capacidad de inspirar, influir y ayudar a desarrollar a otros mientras se manejan los conflictos.

Son estas habilidades específicas las que influyen y persuaden a otros, que impulsan a gestionar y a mejorar el rendimiento de los demás, utilizando y dirigiendo las diversas fortalezas de negociación de un equipo, así como habilidades de manejo de conflicto. (Goleman 2013)

\section{Desempeño laboral}


Inteligencia emocional, el desempeño en ventas y compromiso organizacional Vol. 1, núm. 5., (2017)

Eva María de Lourdes Loaiza Massuh; Jazmin Arce Recalde; Mauricio Vicente Villacreses Cobo

Kumari y Pandey (2011) presentaron al desempeño laboral como aquella actitud que produce consecuencias en varios frentes de evaluación cognitiva, entre ellos están las emociones, las creencias, los comportamientos y el clima laboral en una organización y por ende la conducta organizacional. Mientras que Badir (2015) simplemente definió el desempeño laboral como "la reacción de los empleados hacia su trabajo" (p.65). Por otro lado Shrestha y Baniya (2016), señalaron que la definición de desempeño laboral fue introducida por primera vez en la literatura organizacional en el año 1965 por Lodahl y Kejner, mencionaron que estos autores presentaron el desempeño laboral como la experiencia del grado de absorción que experimenta una persona en su trabajo diario. Más adelante Shrestha y Baniya (2016) también sugirieron que cuando una persona participa de manera activa en su trabajo, es porque percibe el desempeño como el centro de su autoestima y como su auto concepto.

El desempeño laboral ha sido relacionado con el compromiso organizacional observando como el colaborador se desempeña con relación a las constituyentes organizacionales y los parámetros observados radican en la lealtad demostrada, el comportamiento de ciudadanía organizacional, el comportamiento contraproducente, el empeño en su labor particular, satisfacción laboral y otros constructos de índole individual y grupal. Se espera que los empleados comprometidos puedan llegar a desarrollar un mayor nivel de desempeño laboral que aquellos quienes no están comprometidos laboralmente.(Khan et al, 2014)

\section{Desempeño en ventas}

Wiatr y Johnston (2012) conceptualizaron el desempeño en ventas como la evidencia del status profesional llevado a la práctica en el día a día en las calles al vender Brown y Peterson 


\section{Inteligencia emocional, el desempeño en ventas y compromiso organizacional}

Vol. 1, núm. 5., (2017)

Eva María de Lourdes Loaiza Massuh; Jazmin Arce Recalde; Mauricio Vicente Villacreses Cobo

(1994) luego de notar la falta de un rumbo en cuanto a cómo determinar la brecha laboral fundamental entre el rendimiento de ventas y la satisfacción en el trabajo realizaron una investigación llevada a cabo en una organización de ventas directas, en la que propusieron la teoría del valor terminal, que consiste en ampliar las medidas de rendimiento de ventas para incluir la perspectiva de valor psicológico de trabajo, los resultados dejaron ver que el nivel de satisfacción laboral luego de un alto rendimiento en ventas dura mientras duren los réditos recibidos.

Otro modelo presentado fue el de Bagozzi (1978) quien explicó el desempeño, satisfacción laboral y otras medidas de resultado conductuales experimentados por los vendedores. En este modelo "de hombre" el comportamiento de los vendedores se muestra como una función de la persona, las interacciones que la persona tiene con otras personas especialmente dentro de la organización son significativas en su desempeño del rol. También Murray, Stewart y Piotrowski (2002) presentaron un modelo que consistía en auscultar los efectos mediadores de orientaciones de trabajo cognitivo-motivacionales sobre las relaciones entre los rasgos de personalidad y el rendimiento en un trabajo de ventas. No obstante la discrepancia estaba relacionada con la lucha por el buen trato dentro de la organización. El desarrollar de la inteligencia emocional en estos equipos de vendedores pudiera fortalecer estas interacciones con otras personas, elevando el rendimiento laboral y afianzando el compromiso organizacional.

\section{Compromiso organizacional}


Inteligencia emocional, el desempeño en ventas y compromiso organizacional Vol. 1, núm. 5., (2017)

Eva María de Lourdes Loaiza Massuh; Jazmin Arce Recalde; Mauricio Vicente Villacreses Cobo

Amirghodsi y Naeini (2016) definieron el Compromiso organizacional como la tendencia de un individuo a comprometerse y participar en procesos y actividades organizacionales con la comprensión del costo - oportunidad que este implica. Se refirieron también la definición que Porter le dio como la intensidad de la identificación personal de un individuo basada en su organización y participación en el mismo. Por su parte Gholami, Shams, y Amoozadeh (2013) explicaron que el compromiso organizacional puede definirse como la capacidad de reconocimiento del individuo conjugado con actividades en una organización. El compromiso de la organización es un enfoque que establecen los miembros de la organización, en qué medida determinan su propia identidad y cuánto se cuestionan con ella.

Para Becker, Billings y Gilbert (1996) un aspecto de consideración del compromiso organizacional tiene sus raíces en términos de intercambio o nociones de costo-beneficio donde el énfasis está en la negociación entre el individuo y la organización: mientras más favorable es el intercambio, mayor será el compromiso del individuo para con la organización.

Para Meyer y Allen (1991) el compromiso organizacional consiste de componentes como afectividad, continuidad y compromiso con las normativas. Los componentes del compromiso organizacional se caracterizan por mentalidades diferentes y por lo tanto tienen diferentes implicaciones, por ejemplo el compromiso de permanencia puede verse afectado primordialmente por consideraciones externas o como se lo conoce en otros ámbitos el "lado opuesto de las cosas" como prestaciones de jubilación o planes de retiro. Es similar al llamado compromiso organizacional calculado, construido sobre la base de la misma teoría del " lado apuesto de las cosas ". Por su lado Suliman \& Isles (2000) resaltaron luego de su investigación que el acercamiento de lo que sería la conceptualización más acertada de compromiso 


\section{Inteligencia emocional, el desempeño en ventas y compromiso organizacional}

Vol. 1, núm. 5., (2017)

Eva María de Lourdes Loaiza Massuh; Jazmin Arce Recalde; Mauricio Vicente Villacreses Cobo

organizacional incluyó la conducta actitudinal y las dimensiones normativas, fue así como determinaron que el compromiso organizacional es un constructo multidimensional, las características en mención son los elementos que establecen los parámetros bajo los que conforme a lo explicado por Shanker y Sayeed (2015) el compromiso organizacional debe ser medido, esto es: compromiso afectivo, compromiso de continuidad y compromiso normativo.

De acuerdo a la explicado por Khan et al (2014) el compromiso afectivo se relaciona con el apego emocional de los empleados de su organización, compromiso de continuidad se refiere al compromiso de los empleados a la organización, debido al costo de oportunidad percibida de dejar la organización y compromiso normativo se refiere a las normas sociales y valores que afectan el compromiso del empleado con la organización. El compromiso afectivo por ejemplo puede llegar a ser impactado principalmente por experiencias laborales positivas, ayuda a percibir el apoyo organizativo y hasta demostrar sacrificio a favor de la organización.

Están dispuestos a trabajar horas extras cuando el trabajo así lo requiere. También están dispuestos a promover la organización como un lugar propicio para trabajar.

Esta podría ser una medida de la fuerza relativa de la participación de un individuo con una organización. Cuando los individuos tienden a mostrar sacrificio, entonces el compromiso con la organización se vuelve significativo.

\section{La inteligencia emocional, el desempeño en ventas y el compromiso organizacional}

Una cultura organizacional emocionalmente inteligente enfatiza profundamente en la construcción, desarrollo y mantenimiento de relaciones, empatía y responsabilidad social 
Inteligencia emocional, el desempeño en ventas y compromiso organizacional Vol. 1, núm. 5., (2017)

Eva María de Lourdes Loaiza Massuh; Jazmin Arce Recalde; Mauricio Vicente Villacreses Cobo

(Sayeed \& Shanker, 2009). Precisamente estos atributos son los que conforme a lo que Shanker y Sayeed (2015) resaltaron que estos atributos mejoran la confianza y el compromiso además de facilitar la conexión entre un nuevo colaborador contratado y la organización como empleadora.

En una investigación sobre estrategias de trabajo emocional entre profesionales en el campo de las ventas Liu, Prati, Perrewé, y Ferris (2008) comprobaron la hipótesis que los profesionales en ventas con una capacidad emocional definida y bien cimentada, esto es, vendedores que habían desarrollado la inteligencia emocional, mostraron una tendencia más que otros a llevar a cabo actos profundos. Por su parte Devonish, D. (2016) estableció en su estudio que la Inteligencia Emocional hace una importante contribución en los comportamientos del desempeño de los empleados.

También Devonish, D. (2016) desde una perspectiva teórica, estipularon que las proposiciones que rodean la noción y conceptualización de la Inteligencia Emocional han sugerido que empleados con Inteligencia Emocional alta son capaces de modular sus emociones para mejorar su rendimiento en el trabajo, afirmó que: "dentro de un organización, la regulación emocional está teóricamente relacionada con el compromiso organizacional y con el desempeño en el trabajo mediante la inducción de estados afectivos que son beneficiosos para el desempeño laboral" (p.435) . Así mismo Meyer y Allen (1997) concluyeron que los empleados comprometidos son más propensos a permanecer con la organización, trabajar hacia el logro de objetivos organizativos, sobrepasar el cumplimiento de la cuota mensual de ventas, ejercer altos niveles de esfuerzo en nombre de la organización y mostrar la aceptación de valores y objetivos principales de la organización

Revista Científica Mundo de la Investigación y el Conocimiento. 1(5). pp. 578-600 


\section{Inteligencia emocional, el desempeño en ventas y compromiso organizacional}

Vol. 1, núm. 5., (2017)

Eva María de Lourdes Loaiza Massuh; Jazmin Arce Recalde; Mauricio Vicente Villacreses Cobo

También Rode et al (2016) obtuvieron como resultados de su estudio, luego de haber realizado mediciones con el instrumento MSCEIT entre un grupo de 200 vendedores que un alto nivel de Inteligencia Emocional y motivación son necesarios, si lo que se busca es alcanzar un mejor desempeño laboral y fortalecer en los colaboradores el compromiso organizacional. Posteriormente Frye (2012) concluyó que la satisfacción en el trabajo está definida como un estado emocional firme, adquirido por la valoración que se le otorga al profesional de ventas por el trabajo realizado o por experiencias laborales desarrolladas por diversos factores intrínsecos y extrínsecos.

En un campo laboral específico como el de las ventas Deeter-Schmelz (2007) al concluir su estudio respecto de este tema, demostró luego de aplicar varias pruebas como vendedores juniors tanto como seniors que carecen de todos o algunos aspectos de la Inteligencia Emocional pueden ser entrenados para estar emocionalmente mejor alineados con los pensamientos, ideales y necesidades de sus clientes, con los suyos propios y al mismo tiempo para desarrollar el necesario sentido relacional con la organización a la que pertenecen. De la misma manera, Loaiza (2016) citando a Kidwell, McFarland, y Avila (2007) explicó el estudio que llevaron a cabo con la finalidad de establecer la capacidad del vendedor para distinguir las emociones que surgen durante el contacto comercial en un proceso de compra-venta, los resultados revelaron que mientras más alta era la capacidad de percibir las emociones del posible cliente, los efectos eran más beneficiosos en las ventas, pero se dejó la inquietud de buscar en futuras investigaciones nuevas implicaciones al respecto.

Las diferentes conceptualizaciones de lo que implica el desempeño laboral puntualmente en el campo de las ventas, tal como se han presentado anteriormente, así como la 
Inteligencia emocional, el desempeño en ventas y compromiso organizacional Vol. 1, núm. 5., (2017)

Eva María de Lourdes Loaiza Massuh; Jazmin Arce Recalde; Mauricio Vicente Villacreses Cobo

participación en el trabajo no se debe simplemente a las motivos humanas racionales, las emociones de los profesionales también juegan un papel importante que los mueve a involucrarse en sus trabajos y comprometerse con su organización. (Meisler \& Vigoda-Gadot, 2014)

\section{Conclusión.}

El compromiso afectivo se caracteriza por apego emocional, así como la identificación y la participación en la organización; el compromiso normativo se caracteriza por la supuesta obligación a permanecer en la organización y la continuidad del compromiso por el reconocimiento de los costos asociados con dejar la organización (Meyer \& Allen, 1997). Es útil e interesante el tener en cuenta la importancia de la inteligencia emocional para el desempeño laboral eficaz porque la capacidad de una persona para percibir, identificar y administrar su o sus emociones es el origen de las competencias sociales y emocionales que son importantes para el éxito en casi cualquier trabajo. (Meisler \& Vigoda-Gadot, 2014). La inteligencia emocional y el desempeño laboral puntualmente en el campo de las ventas como se ha examinado brevemente en este ensayo, afectan no solo los estados financieros de las empresas, sino también el compromiso organizacional, se recomienda a las organizaciones realizar contrataciones laborales instruyendo al nuevo personal que la base de todo trabajo requiere relación social y ya que la inteligencia emocional es enseñable e incrementable, se recomienda crear un entorno educativo apropiado para capacitar al personal, guiándolos a desarrollar su inteligencia emocional. Además, quienes ocupan cargos gerenciales deben reconocer los factores que afectan el bienestar laboral y tratar de mantener la satisfacción laboral del personal en un nivel deseado porque tanto la inteligencia emocional como el satisfactorio desempeño en el trabajo son dos 


\section{Inteligencia emocional, el desempeño en ventas y compromiso organizacional}

Vol. 1, núm. 5., (2017)

Eva María de Lourdes Loaiza Massuh; Jazmin Arce Recalde; Mauricio Vicente Villacreses Cobo

factores claves que tienen un papel preponderante y un efecto relevante sobre el compromiso organizacional y por lo tanto sobre la eficiencia del rendimiento de la organización.

\section{Bibliografía.}

Alkahtani, A. (2016). The Influence of Leadership Styles on Organizational Commitment: The Moderating Effect of Emotional Intelligence. RedFame, 2(1)23-24. DOI:10.11114/bms.v2i1.1091

Amirghodsi, S., \& Naeini, A. (2016). Cause and Effect Relationships between Emotional Intelligence's Components, Organizational Commitment and Job Satisfaction using Fuzzy DEMATEL Method: Case Study of Iranian Central Oil Fields Company. Mediterranean Journal of Social Sciences,7(452),17-24. Doi:10.5901/mjss.2016.v7n4s2p17

Badir, A. (2015). The Effect of Emotional Intelligence on Job Satisfaction: Applied Study in the Jordanian Telecommunication Sector. International Journal of Business Administration, 6(3), 64-71. doi:10.5430/ijba.v6n3p63

Bagozzi, R. (1978). Salesforce Performance and Satisfaction as a Function of Individual Difference, Interpersonal, and Situational Factors. Journal of Marketing Research,15(4), 517-531. DOI: $10.2307 / 3150622$

Bande, B., Fernández-Ferrín, P., Varela, J., \& Jaramillo, F. (2015). Emotions and salesperson propensity to leave: The effects of emotional intelligence and resilience. El Sevier: Industrial Marketing Management, 44(1), 142-153. DOI.org/10.1016/j.indmarman.2014.10.011

Bar-On, R. (2000). Handbook of emotional intelligence. San Francisco,CA: Jossey-Bass.

Bar-On, R. (2006). The BarOn Model of Social and Emotional Intelligence (ESI) Psicothema, $18(l), 13-25$

Becker, T., Billings, S., Eveleth, D., Gilbert, N. (1996). The mediating effect of job satisfaction between emotional intelligence and organizational commitment of nurses: A questionnaire survey. International Journal of Nursing Studies. 45, 1625-1635. 
Inteligencia emocional, el desempeño en ventas y compromiso organizacional

Vol. 1, núm. 5., (2017)

Eva María de Lourdes Loaiza Massuh; Jazmin Arce Recalde; Mauricio Vicente Villacreses Cobo

Boons, F., Lüdeke-Freund, F. (2013). Business models for sustainable innovation: state-of-theart and steps towards a research agenda. Journal of Cleaner Production, 45(2013), 9-19. DOI.org/10.1016/j.jclepro.2012.07.007

Brown, S., \& Peterson, R. (1994). The Effect of Effort on Sales Performance and Job Satisfaction. Journal of Marketing, 58(2), 70-80. DOI: 10.2307/1252270

Chacón, L. (2013). Propuesta Fundamentada en la Teoría de la Inteligencia Emocional para Fortalecer la Formación Integral de los Comunicadores Organizacionales del Pontificia Universidad Javeriana (Tesis de maestría, Pontificia Universidad Javeriana, Bogotá, Colombia). Recuperado de http://hdl.handle.net/10554/11230

Chernis, C. \& Goleman, D. (20013). Inteligencia Emocional en el Trabajo. Barcelona: Kairós.

Devonish, D. (2016). Emotional intelligence and job performance: the role of psychological well-being. International Journal of Workplace Health Management, 9 (4), 428 - 442. DOI.org/10.1108/IJWHM-04-2016-0031

Deeter-Schmelz D., \& Sojka, J. (2007). Developing effective salespeople: Exploring the link between emotional intelligence and sales performance. The International Journal of Organizational Analysis, 11(3), 211 - 220. doi:http://dx.doi.org/10.1108/eb028972

Del Henar, P. (2013). La inteligencia emocional en el entrevistador periodístico: competencias y formación (Tesis de doctorado. Universidad Católica San Antonio, Murcia, España). Recuperado de http://repositorio.ucam.edu/jspui/bitstream/10952/749/1/Tesis.pdf

Fragoso, R. (2015). Emotional intelligence and emotional competencies in higher education, the same concept?. Science Direct, 6(16), 110-125. DOI:10.1016/j.rides.2015.02.001

Frye., W. (2012). An Examination of Job Satisfaction of Hotel Front Office Managers According to Extrinsic, Intrinsic, and General Motivational Factors. International Journal of Business and Social Science, 3(18),40-52

Gardner, H. (1983). Frames of Mind: The Theory of Multiple Intelligences. New York, NY: Basic Books.

Gholami, B., Shams, S., \& Amoozadeh, M. (2013). The Investigation of the Relationship 


\section{Inteligencia emocional, el desempeño en ventas y compromiso organizacional}

Vol. 1, núm. 5., (2017)

Eva María de Lourdes Loaiza Massuh; Jazmin Arce Recalde; Mauricio Vicente Villacreses Cobo

between Emotional Intelligence. Job Satisfaction and Organizational Commitment of Personnel in Banks and Financial Institutions of Darrehshahr City. International Research Journal of Applied and Basic Sciences, 6(10), 1499-1504.

Gil, J., Suárez, I., \& Pérez, D. (2013). Relación de Causalidad entre los Mercados Emergentes y los Índices Bursátiles (Causation Between Emerging Markets and Stock). CICAG, 1O(1). Recuperado

de http://publicaciones.urbe.edu/index.php/cicag/article/viewArticle/1683/3491

Goleman, D. (1995). Inteligencia Emocional. Barcelona, ES:Romanyá-Valls, S.A.

Goleman, D. (1998), Working with Emotional Intelligence. London, Eng.: Bloomsbury.

Goleman, D., Boyatzis, R. \& Mckee, A. (2002). Primal Leadership: Realizing the Power of Emotional Intelligence, Harvard Business School Press, Boston, MA

Goleman, D., Bennett, L. \& Barlow, Z. (2012). ECO Literatate. San Francisco, CA:Jossey-Bass

Goleman, D. (2013). Primal Leadership. Boston, MS: Harvard Business School Publishing

Gould-Williams, J. (2016). Managers' motives for investing in HR practices and their implications for public service motivation: A theoretical perspective. International Journal of Manpower, 37 (5),764 - 776. DOI:http://dx.doi.org/10.1108/IJM-03-20160065

Jordan, P. J., Ashkanasy, N. M., Härtel, C. E., \& Hooper, G. S. (2002). Workgroup emotional intelligence: Scale development and relationship to team process effectiveness and goal focus. Human Resource Management Review, 12(2), 195-214. DOI: 10.1016S10534822(2)00046-3

Kafetsios, K., \& Zampetakis, A. (2008). Emotional intelligence and job satisfaction: Testing the mediatory role of positive and negative affect at work. Science Direct , 44 (2008), 712-722. Doi:10.1016/j.paid.2007.10.004

Khan, M., Awan, U., Yasir, M., Mohamad, N., Ali, S.,Qureshi, \& M.,Zaman, K.(2014). TRANSFORMATIONAL LEADERSHIP, EMOTIONAL INTELLIGENCE AND ORGANIZATIONAL COMMITMENT: PAKISTAN'S SERVICES SECTOR ARGUMENTA OECONOMICA 2 (33), 67-92. DOI: 10.15611/aoe.2014.2.04

Kidwell, B., McFarland, R., \& Avila, A. (2007). Perceiving Emotion in the Buyer-Seller Interchange: The Moderated Impact on Performance. Journal of Personal Selling \& Sales Management, 27(2), 119-132. Recuperado de http://www.jstor.org/stable/40472099 
Inteligencia emocional, el desempeño en ventas y compromiso organizacional

Vol. 1, núm. 5., (2017)

Eva María de Lourdes Loaiza Massuh; Jazmin Arce Recalde; Mauricio Vicente Villacreses Cobo

Kumari, G., \& Pandey, K.M. (2011). Job Satisfaction in Public Sector and Private Sector: A Comparison. International Journal of Innovation, Management and Technology, 2(3), 7. Recuperado de http://www.ijimt.org/papers/135-M556.pdf

Liu, Y., Prati, L., Perrewé, P., \& Ferris, G. (2008). The Relationship Between Emotional

Resources and Emotional Labor: An Exploratory Study. Journal of Applied Social Psychology, 38(10), 2410-2430. doi: 10.1111/j.1559-1816.2008.00398.x

Loaiza, E. (2016). Inteligencia Emocional en el Desempeño en Ventas. (Tesis doctoral,

CENTRUM, Escuela de Negocios de la Pontifica Universidad Católica de Perú).

Marcano, M. (2006). Estudio de la Inteligencia Emocional de los Empleados de la Empresa Operaciones de Producción y Exploración Nacionales (O.P.E.N.), S.A. (Tesis Doctoral Universidad de Oriente Núcleo de Monagas,Venezuela). Recuperado de http://ri.bib.udo.edu.ve/bitstream/123456789/665/1/TESIS-658.314_M314m_01.pdf

Mayer, J. \& Salovery, P. (1997). What is emotional intelligence?. New York, NY: Basic Books

Mayer, J., Salovey, P., \& Caruso, D. (2004). Emotional Intelligence: Theory, Findings, and Implications. Psychological Inquiry: An International Journal for the Advancement of Psychological Theory,15(3), 197-215. doi:10.1207/s15327965pli1503_02

Meisler, G., \& Vigoda-Gadot, E. (2014). Perceived organizational politics, emotional intelligence and work outcomes: Empirical exploration of direct and indirect effects. Personnel Review, 43(1), 116-135. DOI.org/10.1108/PR-02-2012-0040

Meyer, J. P. and Allen, N. J. (1997). Emotional structure and commitment: implications for health care management. Journal of Health Organization and Management. 19 (2), 120129.

Miranda, E. (2014). El arte de Negociar. El Negociador a Tiempo Completo. Madrid, ES: AIIM(Asociación de Ingenieros Industriales de Madrid)

Murray, B., Stewart, G., Piotrowski, M. (2002). Personality and job performance: Test of the mediating effects of motivation among sales representatives. Journal of Applied Psychology, 87(1), 43-51. http://dx.doi.org/10.1037/0021-9010.87.1.43

Psilopanagioti, A., Anagnostopoulos, F., Mourtou, E., \& Niakas, D. (2012). Emotional intelligence, emotional labor, and job satisfaction among physicians in Greece. BMC Health Serv Res, 12(463), 12-463. DOI: 10.1186/1472-6963-12-463

Rode, J., Mooney, Ch., Arthaud-Day, M., Near, J., Baldwin,T., Rubin, R.\& Bommer, W.

Revista Científica Mundo de la Investigación y el Conocimiento. 1(5). pp. 578-600 


\section{Inteligencia emocional, el desempeño en ventas y compromiso organizacional}

Vol. 1, núm. 5., (2017)

Eva María de Lourdes Loaiza Massuh; Jazmin Arce Recalde; Mauricio Vicente Villacreses Cobo

(2016). Emotional Intelligence and Individual Performance: Evidence of Direct and Moderated Effects.Journal of Organizational Behavior, 28(4), 399-421.

URL: http://www.jstor.org/stable/30162566

Saarni, C. (1990). Emotional competence: How emotions and relationships become integrated. Lincoln, NE: University of Nebraska Press.

Salovey, P., \& Mayer, J. D. (1990). Emotional intelligence. DOI: $10.1080 / 00223891.1990 .9674037$

Sayeed, O.B \& Shanker, M. (2009). Emotionally Intelligent Managers and Transformational Leadership Styles. The Indian Journal of Industrial Relations, 44 (4), 593-610.

Seyedeh, Z., Hoda, M., Forogh, A., \& Azar, P. (2013). Relationship between emotional intelligence and organizational Commitment in Iran's Ramin thermal power plant. ScieVerse Science Direct 84( 2013 ) 815 - 819. DOI: 10.1016/j.sbspro.2013.06.653

Shanker, M. \& Sayeed, O. (2015). Organizational Commitment: Some Linkages with Emotional Intelligence. The Indian Journal of Industrial Relations, 51 (2), 312-326.

Shooshtarian, Z., Ameli, A., \& Lari, M. (2013). The Effect of Labor's Emotional Intelligence on Their Job Satisfaction, Job Performance and Commitment. Iranian Journal of Management Studies (IJMS), 6(1), 27-43.

Shrestha, A., Baniya, R. (2016). Emotional Intelligence and Employee Outcomes: Moderating Role of Organizational Politics. Business Perspectives and Research, 4(1), 15-16.

DOI: $10.1177 / 2278533715605426$

Simons, S. \& Simmons, J. (2005). Cómo Medir la Inteligencia Emocional. Madrid, ES: EDAF, S.A.

Suliman, A.M. \& Isles, P.A. (2000). The Multidimensional Nature of Organizational Commitment in a Now-Western Context. Journal of Management Development, 19(1),71-82.

Thorndike, Edward L., Bregman, E. O., \& Cobb, M. V. (1926). The measurement of intelligence. New York, NY: Teachers College Bureau of Publications. 


\section{Inteligencia emocional, el desempeño en ventas y compromiso organizacional}

Vol. 1, núm. 5., (2017)

Eva María de Lourdes Loaiza Massuh; Jazmin Arce Recalde; Mauricio Vicente Villacreses Cobo

Wechsler, D. ( 1958). The Measurement and Appraisal of Adults Intelligence. Baltimore, MD: Williams \& Wilkins

Wiatr, S., \& Johnston, W. (2012). The IPS-EQ Model: Interpersonal Skills and Emotional Intelligence in a Sales Process. Journal of Personal Selling and Sales Management 33(1), 39-51. doi: 10.2307/23483318 